\title{
In situ evaluation of spatiotemporal distribution of doxorubicin from Drug-eluting Beads in a tissue mimicking phantom
}

\author{
Marcus Caine ${ }^{\star a}$, Shuning Bian ${ }^{\star b}$, Yiqing Tang ${ }^{\star a}$, Pedro Garcia ${ }^{\mathrm{a}}$, Alex Henman ${ }^{\mathrm{a}}$, \\ Matthew Dreher ${ }^{\mathrm{a}}$, Dan Daly ${ }^{\mathrm{c}}$, Robert Carlisle ${ }^{\mathrm{b}}$, Eleanor Stride ${ }^{\mathrm{kb}}$, Sean Willis ${ }^{\mathrm{a}}$, and \\ Andrew L. Lewis*a \\ a Boston Scientific, Lakeview, Watchmoor Park, Camberley, GU15 3YL, UK \\ ${ }^{b}$ Oxford Institute of Biomedical Engineering, University of Oxford, OX3 7DQ, UK \\ c Lein Applied Diagnostics, Reading Enterprise Centre, University of Reading, Earley \\ Gate, Whiteknights Road, Reading, RG6 6BU, UK \\ *Authors contributed equally to this work
}

Corresponding author: Yiqing Tang, Boston Scientific, Watchmoor Park, Camberley GU15 3YL, UK. Email: yiqing.tang@bsci.com

\begin{abstract}
Understanding the intra-tumoral distribution of chemotherapeutic drugs is extremely important in predicting therapeutic outcome. Tissue mimicking gel phantoms are useful for studying drug distribution in vitro but quantifying distribution is laborious due to the need to section phantoms over the relevant time course and individually quantify drug elution. In this study we compare a bespoke version of the traditional phantom sectioning approach, with a novel confocal microscopy technique that enables dynamic in situ measurements of drug concentration. Release of doxorubicin from Drug-eluting Embolization Beads (DEBs) was measured in phantoms composed of alginate and agarose over comparable time intervals. Drug release from several different types of bead were measured. The non-radiopaque DC Bead ${ }^{\mathrm{TM}}$ generated a higher concentration at the boundary between the beads and the phantom and larger drug penetration distance within the release period, compared with the radiopaque DC Bead LUMI TM. This is likely due to the difference of compositional and structural characteristics of the hydrogel beads interacting differently with the loaded drug. Comparison of in vitro results against historical in vivo data show good agreement in terms of drug penetration, when confounding factors such as geometry, elimination and bead chemistry were accounted for. Hence these methods have demonstrated potential for both bead and gel phantom validation, and provide opportunities for optimisation of bead design and embolization protocols through in vitro-in vivo comparison.
\end{abstract}

KEYWORDS Drug-eluting Embolization beads (DEBS), drug distribution, in vitro-in vivo correlation, confocal microscope. 


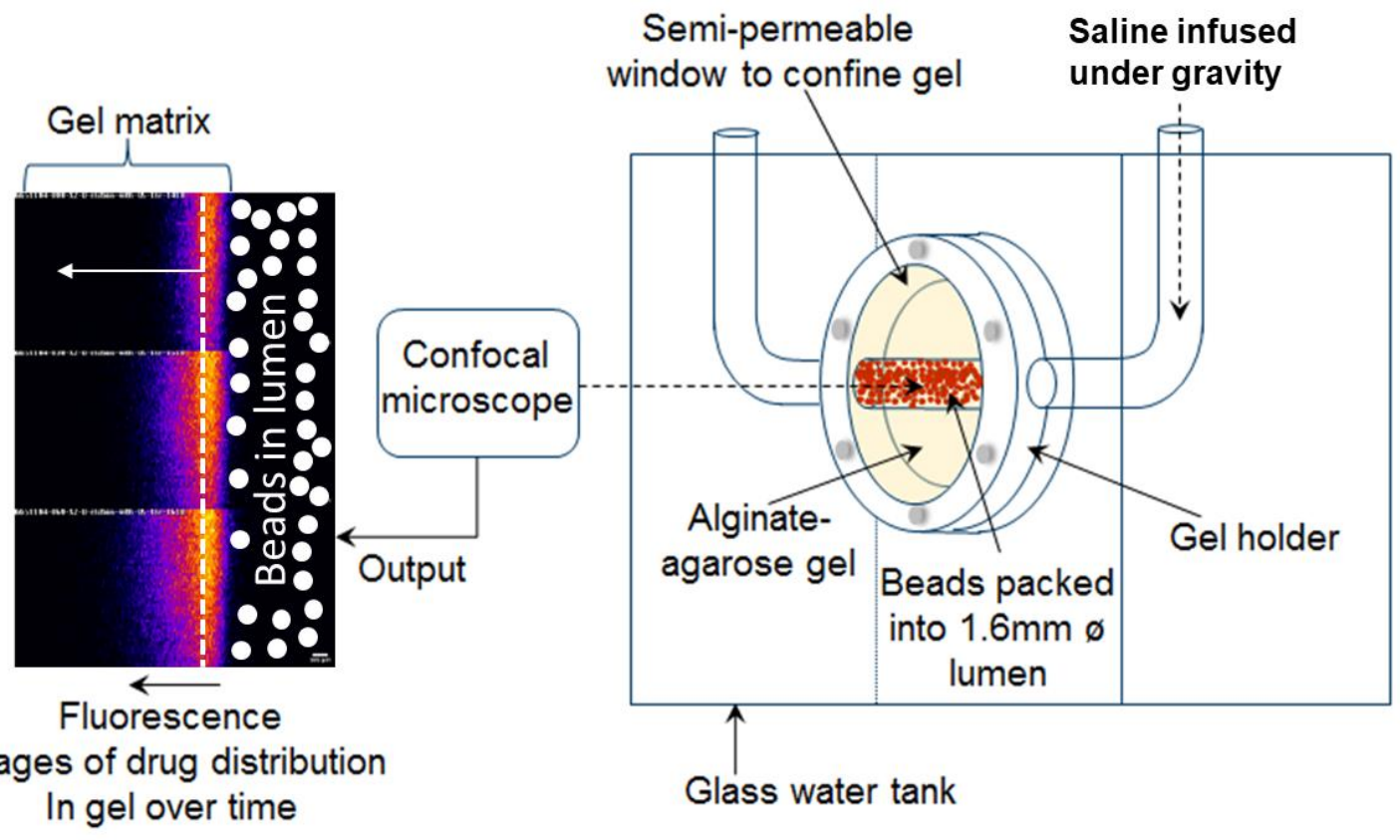




\section{Introduction}

Drug-eluting embolization Beads (DEBs) now play an important role in the treatment of several liver malignancies in hospitals worldwide due to their cost-effectiveness, ease of use and reproducible clinical outcomes [1-3]. DEBs are administered using a minimally-invasive image-guided procedure. They are injected into the hepatic blood supply via a microcatheter and carried into the tumor vessels where they physically occlude the flow, starving the tumor of vital oxygen and nutrients. A chemotherapeutic agent loaded within the bead structure is then released over time into the local environment, at a rate that depends upon a variety of factors including the strength of drug-bead interactions, bead size, number of beads packed into a vessel, dose of drug, drug physicochemical characteristics and nature of the surrounding tissue [4].

DEB elution has traditionally been characterized using flow cells designed to emulate the processes of ion-exchange, diffusion and convection in vivo and hence the likelihood of any systemic toxicity prior to human trials [5, 6]. Although useful for assessing burst elution under flow (i.e. amount of drug released systemically), these methods do not allow for mimicking the in situ kinetics of drug distribution directly into the tissue. It is these kinetics, however, that give a stronger indication of the local efficacy of the treatment [7]. Until recently, information on local levels of drug in the tissue have been obtained only in costly and laborious animal models of liver cancer (including rat, rabbit and woodchuck), or from healthy large animal hepatic arterial embolization studies [8-10]. There has, however, been significant progress in the use of microfluidic technologies for vasculature biomimicry to generate both twodimensional and complex three-dimensional biomimetic in vitro models using cellladen hydrogels [11]. Whilst the focus for these models has been largely towards organ-on-a-chip development and study of cellular processes [12], little has been described for studying arterial embolization and drug delivery. We have presented previously both a microfluidic device that replicated three levels of vessel branching [13] and a silicon-based vascular flow simulator with a twenty-branch architecture $[14,15]$ that have been used to evaluate drug-elution from embolic microspheres. Both models, however, have significant limitations, not least because they are unable to model the diffusion of a drug from an embolic device into the surrounding tissues. Most recently an ex vivo decellularized rat liver model has been described with preserved liver-specific vasculature and optical transparency, that has been used to evaluate both liquid and particulate embolization agents [16]. Whilst the complex vessel structures are maintained, the model still lacks the element of cellular uptake and metabolism and is complex and challenging to use as a routine screening method for product development purposes.

Given the widespread integration of hydrogels in microfluidic applications due to flexibility of preparation, free diffusion of small molecules, biocompatibility, potential for temperature sensitivity and low cost [17], we have been motivated to develop a 
hydrogel tissue-mimicking model into which DEBs can be implanted and drug diffusion kinetics monitored. In the present study we compare differences in drug release from DEBs with different chemical structures, using both a physical sectioning method and a non-invasive in vitro method utilising confocal microscopy, that have been developed in independent laboratories with the intent to interrogate drug elution into tissue mimics in real-time. The drug release measured with each method and bead type are then analysed and compared to historical in vivo imaging data conducted in a swine implantation model to determine the potential of the methods as relatively fast and facile techniques to predict drug diffusion in tissue from DEBs.

\section{Materials and methods}

\subsection{Materials}

Sodium alginate (A1112, low viscosity grade) and agarose (A9535, BioReagent) were purchased from Sigma (St Louis, MO, USA). Doxorubicin hydrochloride powder (Dox) was purchased from Hisun Pharmaceuticals (Zhejiang, China) at $99 \%+$ purity. Embolic beads used in this study were 70-150 $\mu \mathrm{m}$ DC Bead M1 ${ }^{\mathrm{TM}}$ (DC Bead) and its radiopaque counterpart, 70-150 $\mu \mathrm{m}$ DC Bead LUMI ${ }^{\mathrm{TM}}$ (LUMI), both provided by Biocompatibles UK Ltd (Farnham, UK). Saline solution $(0.9 \% \mathrm{w} / \mathrm{w})$ was purchased from Fresenius Kabi AG (Homburg, Germany) and water for injection (WFI) (Baxter, UK). Deionised water of Milli-Q quality was prepared from a Satorius Arium Comfort system (Goettingen, Germany).

\subsection{Drug-eluting Bead (DEB) preparation}

Dox was dissolved in deionized water to produce a stock solution of $25 \mathrm{mgmL}^{-1}$ concentration. Both DC Bead and LUMI were loaded with Dox to obtain a dose of $37.5 \mathrm{mgmL}^{-1}$ by firstly removing the majority of the saline solution in which they were received to leave a $2 \mathrm{~mL}$ wet slurry of beads in the vial, to which was added $3 \mathrm{~mL}$ of the Dox stock solution ( $75 \mathrm{mg}$ total dose). This was left for 1 hour with occasional agitation to load the beads completely with drug (turning the beads red and the loading solution very pale pink indicating $>99 \%$ loading was achieved).

\subsection{Dox distribution by sectioning method}

A mixed agarose (1.5 wt\%) and alginate (0.5 wt\%) hydrogel was used as the tissue mimic with the expectation that Dox diffusion would be determined by the chargecharge interaction between Dox and carboxylic acid on the alginate [18]. To prepare the hydrogel, sodium alginate was dissolved in saline to make a $2 \mathrm{wt} \%$ solution. This was roller-mixed over $24 \mathrm{hr}$ at room temperature, and then passed through a $5 \mu \mathrm{m}$ filter to remove any undissolved particles. A 2 wt\% agarose solution was prepared 
separately by dissolving agarose into saline at $95^{\circ} \mathrm{C}$ first, then the temperature was allowed to fall to $\sim 50^{\circ} \mathrm{C}$ before it was mixed with alginate solution.

The mixed hydrogel was prepared by mixing the appropriate volumes of alginate and agarose solution at $50^{\circ} \mathrm{C}$. This was then cast into cells adapted from $5 \mathrm{~mL}$ syringes and allowed to cool to ambient temperature. A channel with $1.6 \mathrm{~mm}$ diameter was created in the centre of each sample to mimic a blood vessel, and later DEB were packed into this channel (see Fig 1B). The samples were kept in saline until required for the tests described below.

To measure the Dox distribution in the hydrogel over time, the aforementioned gel phantom was packed with Dox-loaded DEBs, by loading beads into the $1.6 \mathrm{~mm}$ diameter channel centred in the gel. Once each channel was fully packed with beads, the syringe tip ends were removed and the gels were gently pushed forward and blade-sectioned using sectioning equipment made in-house. At predetermined time points, $1 \mathrm{~mm}$ thick sections were taken to sample Dox distribution in the hydrogel around the lumen and images were taken with an Olympus SZX16 fluorescence microscope fitted with SC50 camera (2.5x mag with GFPA filter) according to the Dox excitation and emission wavelength range (480 $\mathrm{nm}$ and 600 $\mathrm{nm})$. A CMOS detector with a working distance of $15-20 \mathrm{~mm}$ was employed to monitor drug diffusion from the beads.

The images were processed to obtain the grayscale intensity gradient along the diffusion direction. The captured images were analyzed by drawing a line profile (3-4 $\mathrm{mm}$ ) from the interface between beads and gel lumen to obtain the amplitude of the intensity as a function of distance from the lumen wall (Fig 1B), followed by processing with a smooth function by software Olympus Stream Essentials V2.2. Then the grayscale values were converted to Dox concentration by comparison with a standard curve (see Fig S3 in Supplementary Information) obtained from $1 \mathrm{~mm}$ thick agarose gel sections containing known Dox concentrations.

\subsection{In situ confocal microscopy}

A long working distance $(51 \mathrm{~mm})$ confocal microscope was previously developed to study drug diffusion in tissue under ultrasound excitation [19]. In the present study, this system was used to monitor diffusion of Dox in gel samples in situ, without the need for sectioning. In order to maintain the consistency of fluorescence measurements, the instrument sensitivity was kept constant by fixing the photomultiplier tube (PMT, H9306-04; Hamamatsu Photonics, JP) control voltage at $600 \mathrm{mV}$ and the laser forward current at $60 \mathrm{~mA}$, with the latter corresponding to 0.73 $\mathrm{mW}$ of optical output power. Additionally, multisampling was used to increase instrument sensitivity whilst reducing the signal-to-noise ratio. Optical sections of the phantoms were generated by scanning along the $X, Y$, and $Z$ axes using motorized linear stages. 
Again, a $0.5 \%$ alginate : $1.5 \%$ agarose gel mimic was used with a single $1.6 \mathrm{~mm} \mathrm{ID}$ circular cross-section channel set into the gel contained with a semi-permeable Mylar ${ }^{\circledR}$ window [1] (Fig 1). The Dox-loaded DEB were packed into the lumen of the channel (shown in Fig $1 \mathrm{~A}$ ) and sealed with glass beads to prevent movement at both ends when mounting in the confocal system, and $0.9 \%$ saline was allowed to flow through the DEB to replace the solution in the lumen for $30 \mathrm{~min}$ before confocal microscopy measurement. The gel was then mounted inside a tank filled with deionised and degassed water in front of the confocal laser source and detector. Instrument parameters were optimized to 2 min scan intervals for 1 hour to obtain the maximum dynamic response to fluorescence signals whilst minimising photobleaching of calibration gels preloaded with doxorubicin at various concentrations between 20-50 $\mathrm{MM}$ (Fig S2, Table S1). A diffusion profile and maximum drug penetration distance was reported for each bead type. The temperature of the water bath was kept at room temperature $\left(24^{\circ} \mathrm{C}\right)$.

\subsection{Drug distribution model and data processing}

In a biological setting, the process of drug release from the DEB packed lumen into the surrounding tissue consists initially of ion-exchange between various salts and Dox [20-25]. This is followed by drug diffusion through the bead matrix, then the arterial wall and finally the surrounding tissue; the latter being accompanied by drug metabolism within the cells and elimination by leakage into neighbouring vessels. In this study, the focus was the release of Dox in tissue mimicking gel, taking account of variations due to bead composition, structure and measurement technique.

In this study, a model based on Fick's second law in cylindrical coordinates was used to describe Dox transport in tissue mimicking gel (Eq. 1). As shown in Fig 1, the DEB packed vessel can be modelled as a uniform cylinder, containing fluid that is static once embolization is achieved. It is also assumed that the lumen wall is the integrated part of the cylinder without further differentiation.

$$
\frac{\partial c}{\partial t}=\frac{D}{r} \frac{\partial}{\partial r}\left(r \frac{\partial c}{\partial r}\right)-k c
$$

where $c$ is Dox concentration in $\mu \mathrm{M}, r$ is distance along the radial direction in $\mathrm{cm}, D$ is the effective diffusion coefficient of Dox in $\mathrm{cm}^{2} \mathrm{~s}^{-1}$, and $k$ is the apparent drug elimination coefficient in $\mathrm{s}^{-1}$.

The boundary conditions are:

$$
\begin{aligned}
& c(R, t)=C \\
& c(L, t)=0 \quad(L \gg R)
\end{aligned}
$$


where $R$ is the radius of DEB packed lumen, $C$ is the maximum drug concentration at the interface of DEB and gel, $L$ is the radius of the gel.

The initial condition is:

$$
c(r, 0)=0
$$

The partial differential equation (1) was solved by numerical analysis using the pde function in Matlab (Mathwork, MA USA). In the case of diffusion in tissue-mimicking hydrogel, the drug elimination coefficient $k=0$, as no metabolism occurred. The radius of the DEB-packed lumen was fixed as $800 \mu \mathrm{m}$. Typical drug diffusion measurements are shown in Figs. 2 and 3 . The average gray values from the edge of the gel were used as the baseline at the initial stage of diffusion. At the later stage of approximately $2 \mathrm{hr}$, the front of drug diffusion reached the edge of gel, so that the distribution of drug concentration deviated from ideal diffusion due to edge effects, and background gray values measured from a bland gel were adapted for calculations. The unknown characteristic parameters, Dox diffusivity $D$ and boundary concentration $C$ were obtained by fitting the data measured from both sectioning method and confocal method by solving equation (1). It is started by an assignment of $D$ and $C$ with estimated values, followed by resolving the numerical solution of equation (1). The calculated $C$-distance profile was compared with experiment data and the sum of the least squares of the difference was calculated out. The process was iterated until a best fit was found by minimising the sum of least squares, and corresponding $D$ and $C$ were determined at the same time.

For fitting of in vivo fluorescence data, where $k \neq 0$, the drug concentration $C$ at the lumen boundary was used directly from measurement data. Dox elimination coefficient $k$ was fixed by using a literature value which was obtained from rat liver tissue [31], to avoid the correlation between multiple fitting parameters. Only the effective diffusivity $D$ was fitted in solving Eq. (1). In the case where a single bead and surrounding tissue were mapped to acquire Dox distribution, the geometry of packed beads was still approximated as a cylinder. The radius of the cylinder was an average of $50 \mu \mathrm{m}$ for calculation purposes. To further estimate the drug release for the in vivo situation, based on the fitted effective diffusivity $D$, the distribution of Dox concentration was calculated in a hypothesis case of $k=0$, which provided total drug released at the time point, including the eliminated drug. By summing the cylindrical drug distribution at $k=0$, the dose of released drug at different times could be calculated from Eq. (5), and a cumulative drug elution profile could be generated.

As shown in Fig 1B, the DEB packed vessel was treated as a uniform cylinder, containing fluid that is static once embolization is achieved. It is also assumed that the lumen wall is the integrated part of the cylinder. Based on the drug distribution profile obtained from the measurement, the amount of released drug $\left(M_{t}\right)$ at different time points could be obtained by summing the quantity of drug in a cylindrical volume along its radial direction: 


$$
M_{t}=\sum_{i} \pi l\left(r_{i+1}^{2}-r_{i}^{2}\right) \frac{\left(C_{i}+C_{i+1}\right)}{2}
$$

where $I$ is the length of DEB-packed lumen, $r_{i}$ is the radius of the selected region obtained directly from distance points converted from pixel, and $C_{i}$ is the drug concentration at each point. The drug release profile could be obtained in the form of $M_{t}$ vs. time to further illustrate the characteristics of drug release from the DEB in an embolized state.

The cumulative drug elution was calculated by summing up the quantity of released drug from the vessel surface to the points in which drug concentration decreased to zero along radial direction. As there is no drug elimination occurred in alginateagarose hydrogel, the amount of drug calculated is the total amount of drug released from beads at the time that fluorescence images were taken, hence the values are used directly as the data points to construct the cumulative drug release profile (see Section S4, Supplementary Information).

The semi empirical power law:

$$
M_{t} / M_{\infty}=a t^{b}
$$

was used to fit the drug release data, where the $M_{t}$ is a cumulative dose released at time $t, M_{\infty}$ is the cumulative dose released at infinite time, which was approximated to the total drug loading, and $a$ is a constant reflecting the structural and geometric characteristics of the delivery system, and $b$ is a release exponent of the value of which is related to the underlying mechanism of release [26]. Exponent $b$ was fixed as 0.45 to represent the packed vessel as a cylinder in the fitting. The fitting results are treated as indication only for the time period corresponding to the experiment and do not predict the elution trend over longer times. They also do not reflect the release mechanism of the packed beads through ion-exchange [23, 27] and the interaction of beads and hydrogel components.

\subsection{Historical in vivo comparative data}

Previously unpublished data conducted as part of a historical swine liver and kidney embolization study described by Dreher et al. [28], was utilised to evaluate the predictive capabilities of both methods. In this study drug loaded DC Bead was delivered in a healthy swine model (kidney and liver) and drug release was then quantified by epi-fluorescence sectioning of the surrounding tissue following sacrifice. This was conducted at a single and multiple bead level and showed the tissue distribution of Dox including elimination in vivo (see Section S4, Supplementary Information).

\section{Statistics}


ANOVA and t-Test analyses were performed in Excel (Microsoft) by comparing two groups of data. $\mathrm{P}$ values less than 0.05 were considered to indicate statistical significance, and all statistical tests were two-sided. Data are reported as means with SD unless otherwise indicated.

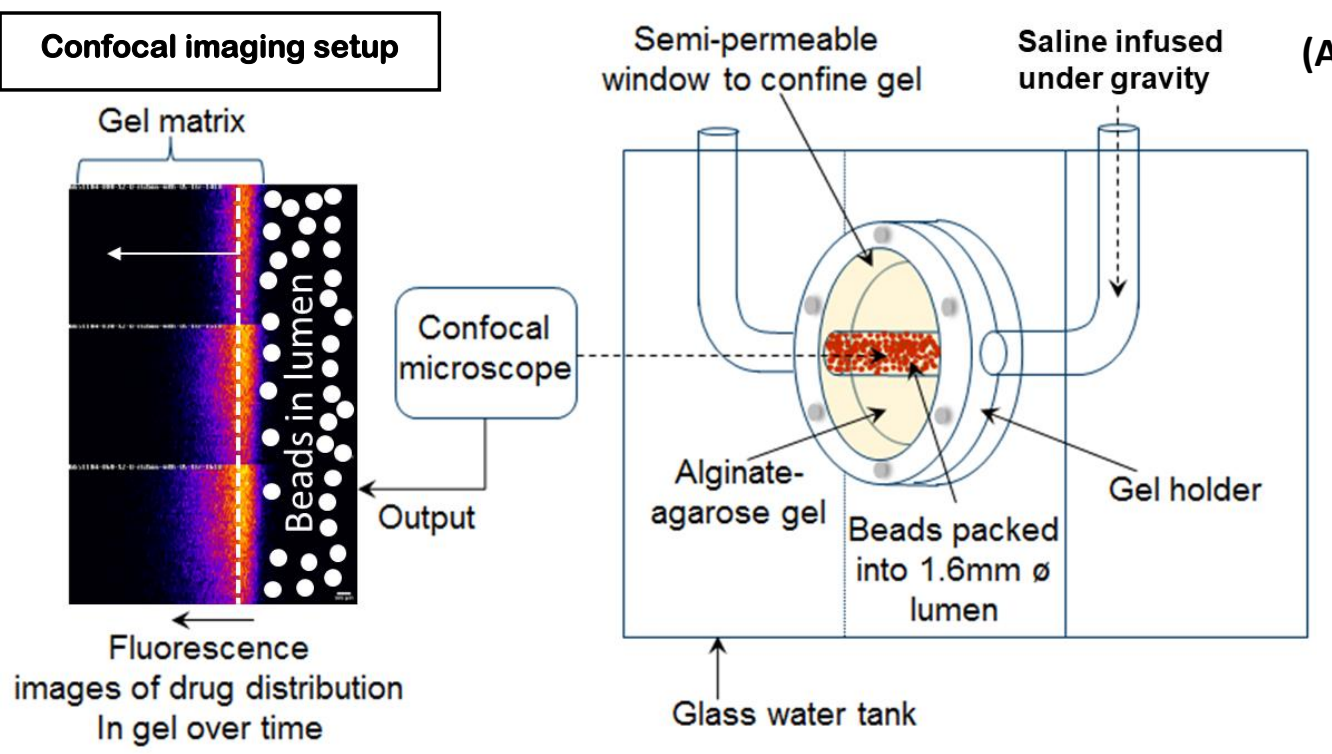

(A)

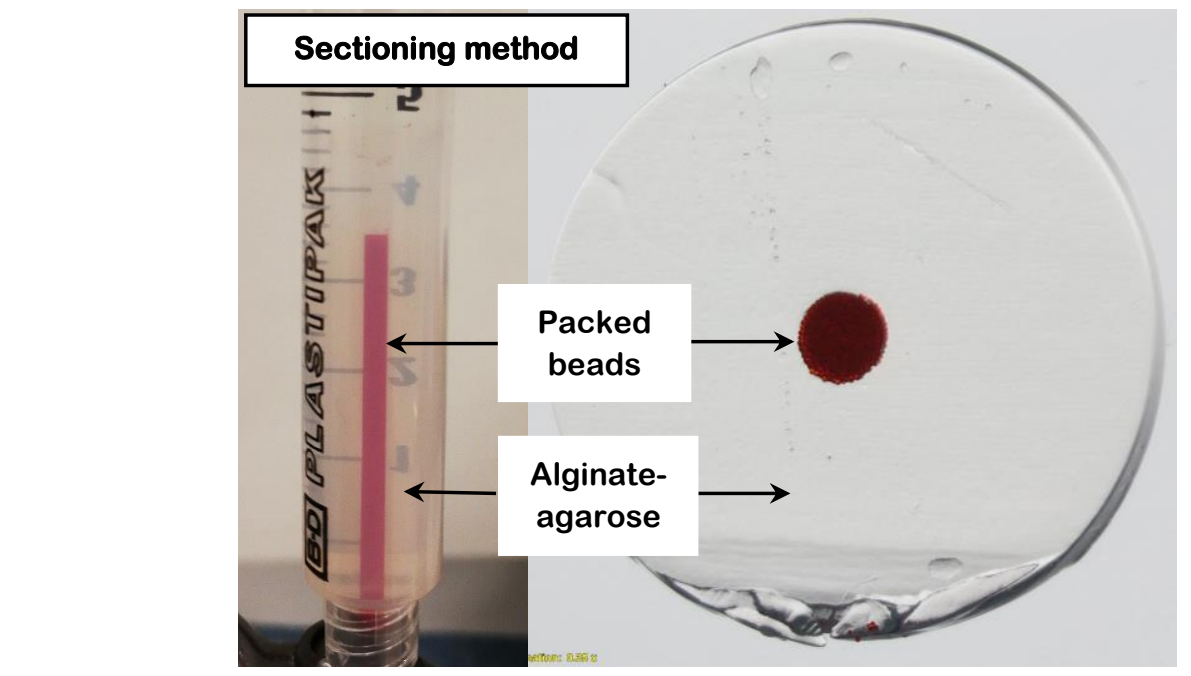

(B)

Figure 1: (A) Experimental setup for confocal fluorescence measurement including mounted agarose: alginate gel matrix immersed in a water bath at room temperature. With false colour rendering of the fluorescent signal output over time in the gel matrix. (B) Left picture is gel sample with a $1.6 \mathrm{~mm}$ ID channel of packed Dox-loaded beads. The central picture shows a sectioned gel in $1 \mathrm{~mm}$ thickness.

\section{Results and discussion}

\subsection{Effect of DEB chemical structure on Dox distribution profiles}


The fluorescence measurement of Dox elution and diffusion from both DC Bead and LUMI within agarose $(1.5 \%)$-alginate $(0.5 \%)$ gel phantoms are shown in Fig. $2 \mathrm{~A}-\mathrm{F}$. A direct visual comparison between DC Bead and LUMI indicates that Dox covered much larger area in DC Bead samples (A-C) than that of LUMI (D-F), suggesting that drug release from $D C$ Bead was much faster. The extracted distribution profile of the Dox concentration and fitted curve by solving Eq. (1) were shown in Fig. $2 \mathrm{G}$ and $2 \mathrm{H}$. The fitted parameters of diffusivity and Dox concentration at interface between beads and gel wall were listed in Table 1. Both the values of penetration of drug front and fitted interface drug concentration of DC Bead are higher than that of LUMI at each time points. The difference in penetration distance is not marked as peak concentration, only about 300-500 $\mu \mathrm{m}$ further in the case of DC Bead.

Fig. 3A-F are the images of in situ measurement of confocal microscopy, which demonstrated the immediate acquirement of drug distribution in both radial and axial direction. From the images, the Dox diffusion in gel along the axial direction were not uniformly distributed, a reflection of gradual bead loading process when sample was injected into the vessel. Similar to what observed in sectioning method, Dox released from DC Bead covered more area, and higher concentration of drug was measured as shown in yellow and orange colour. A conversion of the intensity into concentration and fitted curves are shown in Fig. 3G-H, and the fitted diffusivity and Dox concentration at interface are listed in Table 1. Comparing the penetration front of drug release indicated that the Dox diffused much further in DC Bead sample, almost more than $1000 \mu \mathrm{m}$ more than LUMl sample at $150 \mathrm{~min}$.

Table 1. Fitted diffusivity in hydrogel and Dox concentration at interface of bead packed vessel *

\begin{tabular}{lccc}
\hline \multicolumn{1}{c}{ Sample entries } & $\begin{array}{c}\text { Sampling time } \\
\text { range }(\mathrm{min})\end{array}$ & $\begin{array}{c}\text { Diffusivity } \\
\left(\mathrm{cm}^{2} \mathrm{~s}^{-1}\right)\end{array}$ & $\begin{array}{c}\text { Dox conc. at interface } \\
(\mu \mathrm{M})\end{array}$ \\
\hline DC Bead (sectioning) & $10-120$ & $4.58 \pm 1.24 \times 10^{-6}$ & $98.9 \pm 22.9$ \\
LUMI (sectioning) & $10-120$ & $4.31 \pm 1.33 \times 10^{-6}$ & $36.3 \pm 3.7$ \\
DC Bead (confocal) & $30-150$ & $1.33 \pm 0.67 \times 10^{-6}$ & $1184.8 \pm 134.1$ \\
LUMI (confocal) & $30-150$ & $6.07 \pm 1.85 \times 10^{-7}$ & $865.1 \pm 105.7$
\end{tabular}

\footnotetext{
* The data were presented in average values \pm standard deviation
} 

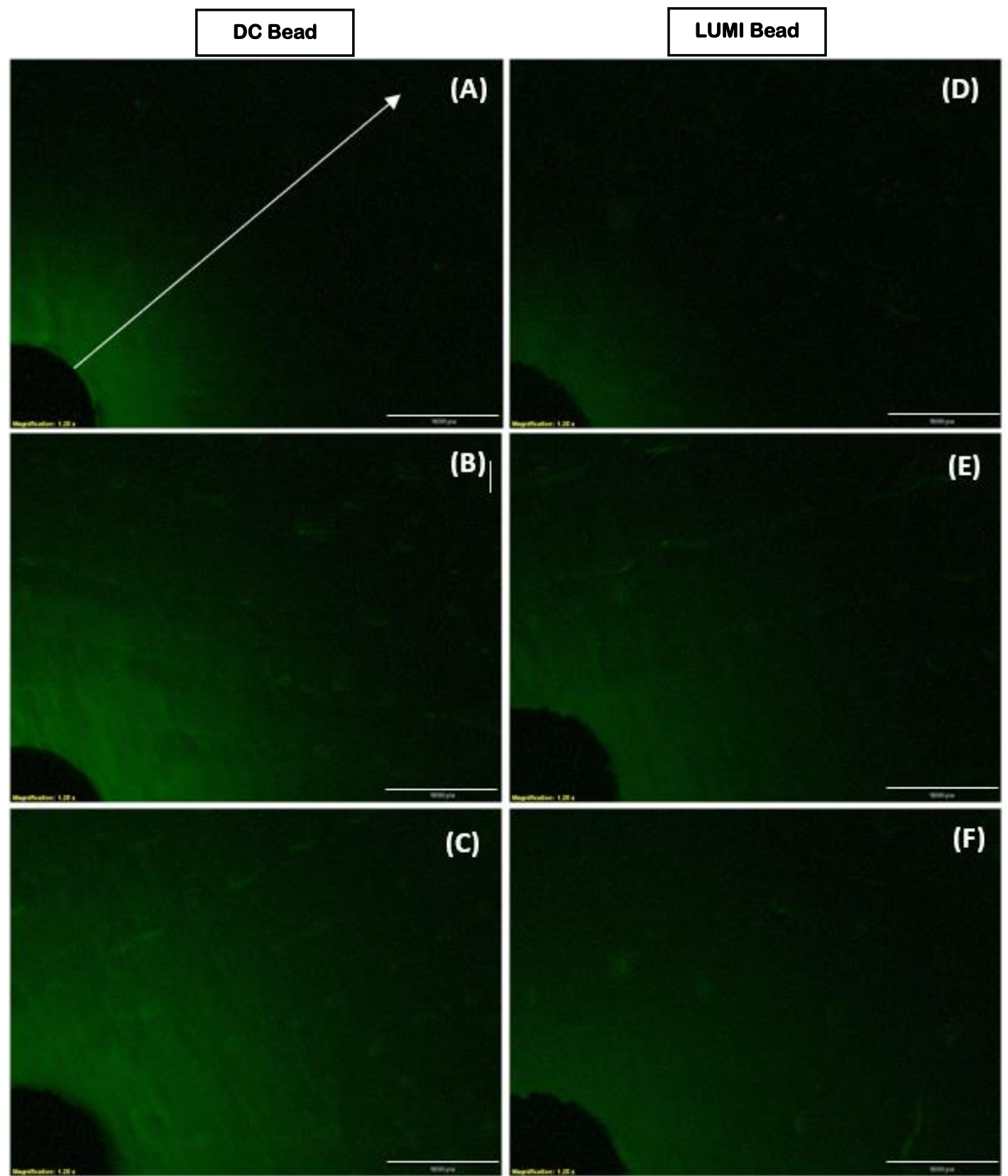

361

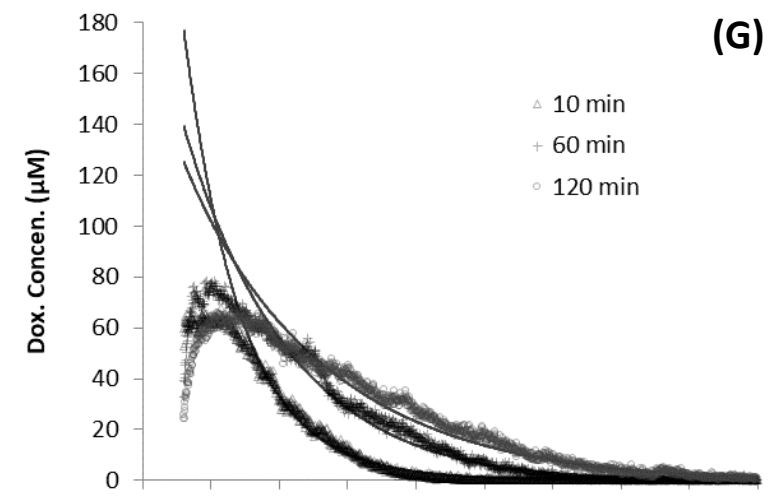

500100015002000250030003500400045005000 Distance from vessel centre $(\mu \mathrm{m})$

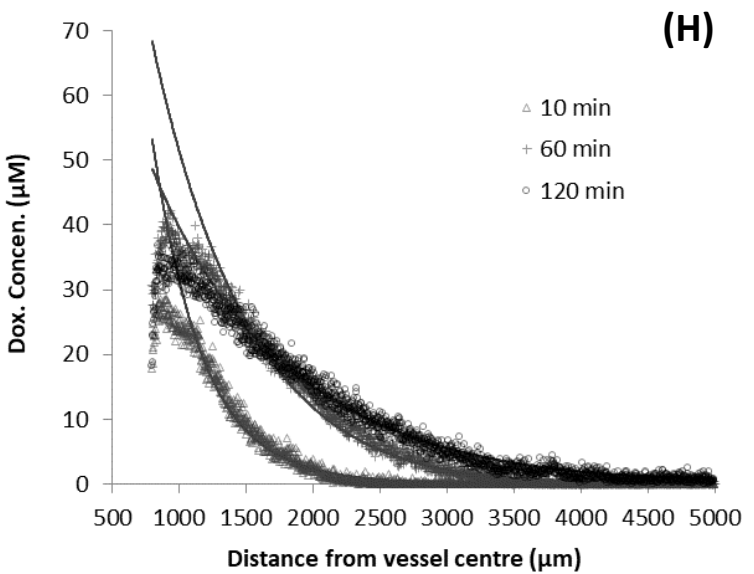


Figure 2. Fluorescence measurement of Dox release from DC Bead and LUMI in hydrogels composed of agarose $(1.5 \%)$-alginate $(0.5 \%)$ obtained by manual sectioning gel samples. (A) Representative fluorescence image of Dox released at $10 \mathrm{~min}$, (B) $60 \mathrm{~min}$ and (C) 120 min from DC Bead. (D) Dox released at $10 \mathrm{~min}$, (E) $60 \mathrm{~min}$ and (F) $120 \mathrm{~min}$ from LUMI. The brightness of the images was increased by $30 \%$ for visual guidance only. (G) Concentration distribution of Dox vs. distance from vessel centre calculated from measurement $(A)-(C)$ of DC Bead based on original images. Solid lines are fitted from equation (1) where $k=0 .(H)$ Concentration distribution of Dox calculated from measurement (D)-(F) of LUMI based on original images. Solid lines are fitting data. Scale bar is $1000 \mu \mathrm{m}$.

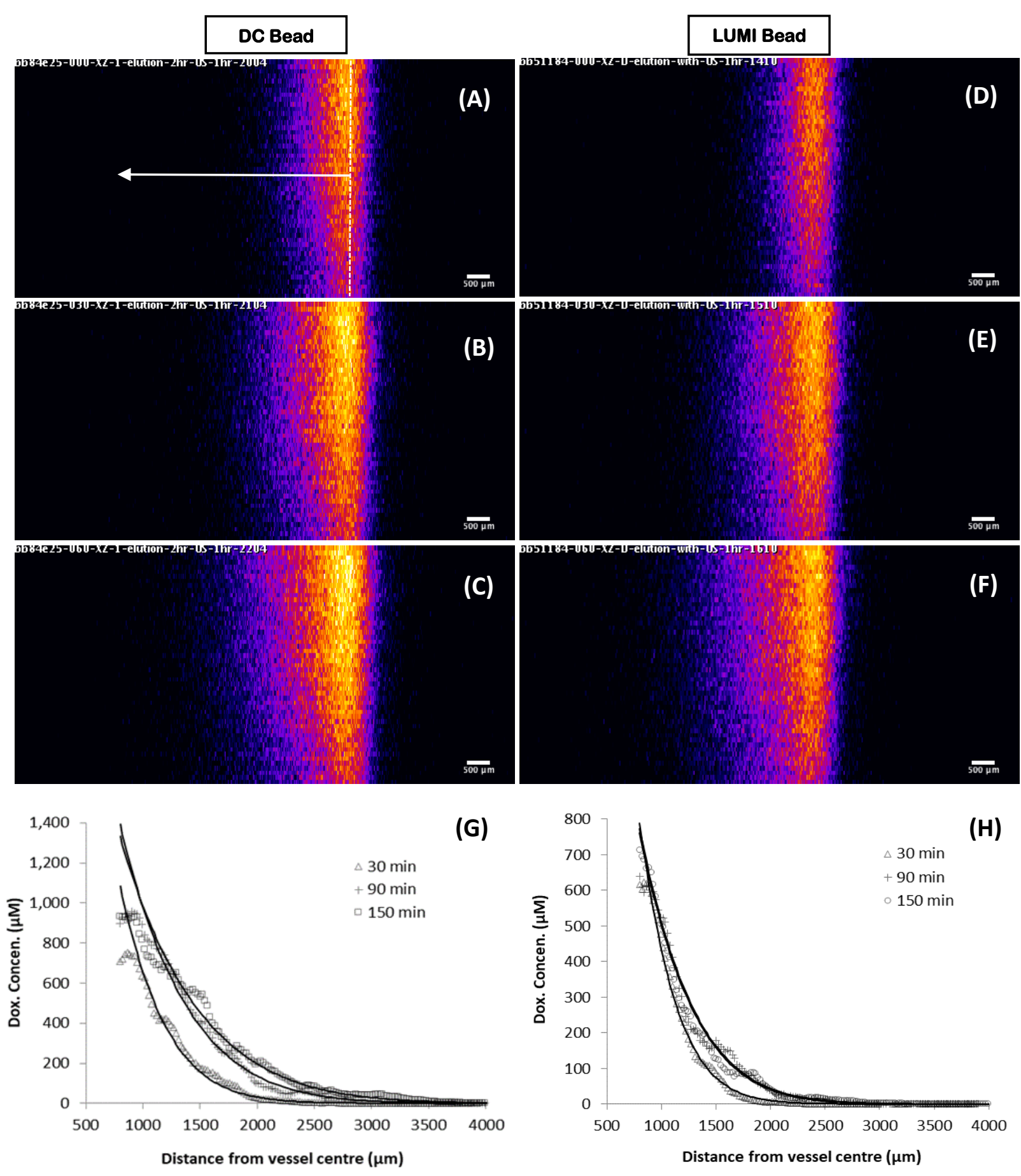


Figure 3. Confocal fluorescence measurements of Dox release from DC Bead and LUMI in hydrogels composed of agarose (1.5\%)-alginate (0.5\%). (A) Representative fluorescence image of Dox released at $30 \mathrm{~min}$ (B) $90 \mathrm{~min}$ and (C) $150 \mathrm{~min}$ from DC Bead. (D) Dox released at $30 \mathrm{~min},(E) 90 \mathrm{~min}$ and (F) $150 \mathrm{~min}$ from LUMI. (G) Concentration distribution of Dox vs. distance from vessel centre calculated from measurement $(A)-(C)$ of DC Bead. Solid lines are fitting data based on equation (1) where $k=0$. $(\mathrm{H})$ Concentration distribution of Dox calculated from measurement (D)-(F) of LUMI. Solid lines are fitting data. Scale bar is 500 $\mu \mathrm{m}$.

Several characteristics were observed with both methods: 1). The drug concentration at boundary of DC Bead was higher than that for LUMI, which is consistent with the fact that DC Bead elutes faster when compared to LUMI [6]; 2). The drug concentration at the boundaries showed almost no change over the $2 \mathrm{hr}$ test period. The second point suggests that the drug release from both DC Bead and LUMI maintained a steady state for at least 2 hrs under the embolized static condition.

\subsection{Drug release measured by confocal and sectioning methods}

By assuming uniform drug release from a packed channel with unit length into the surrounding hydrogel, the total released dose can be calculated by numerical integration (Fig. 4). The cumulative drug release from confocal measurement was 10 times higher than that from the sectioning method in which the DC Bead released $5.7 \mu \mathrm{g}$ of drug and LUMI $2.1 \mu \mathrm{g}$ in $2 \mathrm{hrs}$. The much higher release in the confocal test could be attributed to the procedure of sample preparation, as mentioned above. According to Eq 6., the fitted parameter $M_{\infty} a$ is 0.230 for DC Bead (confocal method), 0.095 for LUMI (confocal method), 0.046 for DC Bead (sectioning method) and 0.017 for LUMI (sectioning method), respectively. The correlation coefficients of power law fitting with the experimental results were in the range of $R^{2}=0.90-0.99$ (Table 2), which suggest the release of drug from a vessel packed with beads followed the trend of power law, without considering elimination effects. A previous study of Dox release from DC Bead (500-700 $\mu \mathrm{m})$ utilising a custom elution device also fitted using a similar equation, in which $b$ was fixed to $0.5[7,29]$. A Level-A correlation between experiment and in vivo results was obtained in this previous study, demonstrating the capabilities of the system to predict release in vivo. 
421

422

423

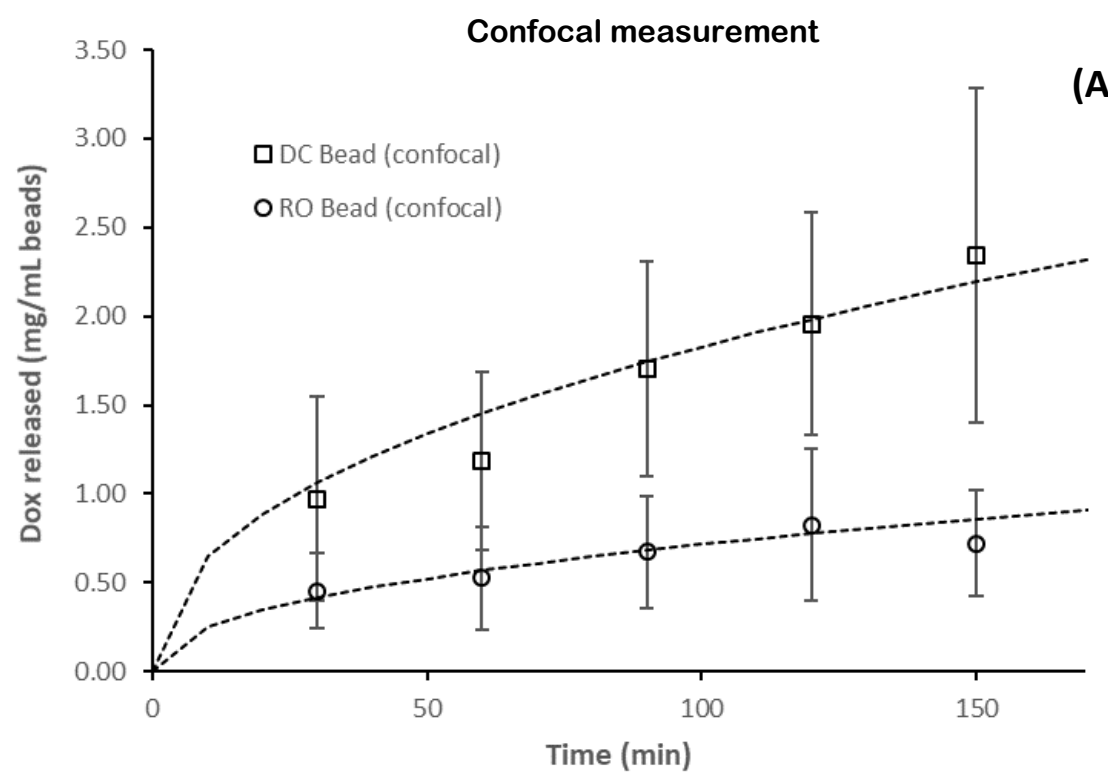

(A)

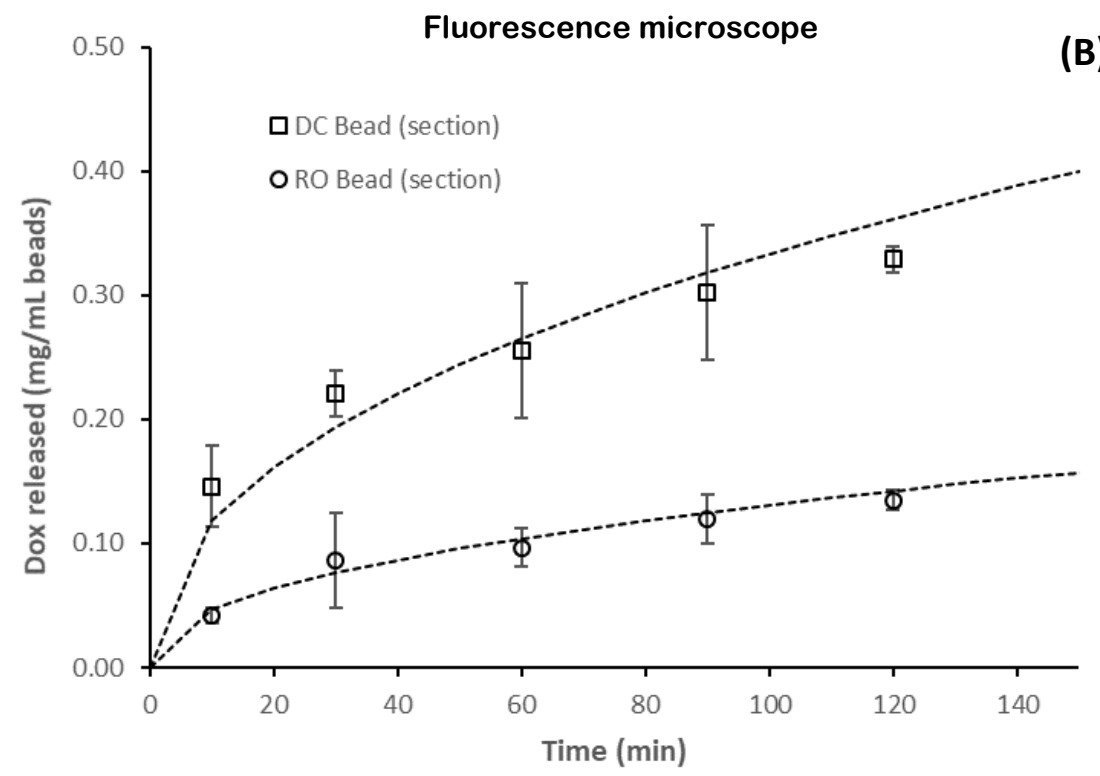

(B)

Figure 4. Dox elution in $\mu \mathrm{g}$ per unit length of vessel based on concentration distribution. (A) confocal fluorescence measurement. (B) fluorescence microscope measurement of sectioned samples. Although the dotted and dashed line were presented as fitted results by power law based on the short period release, it should be treated as indication only in terms of predicting elution trend in long term scale. (Error bars are $\mathrm{SD}, \mathrm{n}=3$ )

Table 2. Parameters for power law fitting of drug release from bead packed vessel

\begin{tabular}{lccccc}
\hline \multicolumn{1}{c}{ Sample entries } & $\begin{array}{c}\text { Bead size } \\
\text { range } \\
(\mu \mathrm{m})\end{array}$ & $\begin{array}{c}\text { Dox dose in } \\
\text { packed vessel } \\
(\mathrm{mg})\end{array}$ & $\begin{array}{c}M_{\infty} a \\
\left(\mathrm{mg}^{*} \mathrm{~min}^{-1}\right)\end{array}$ & $\begin{array}{c}a \\
\left(\mathrm{~min}^{-1}\right)\end{array}$ & $\begin{array}{c}\text { Correlation } \\
\text { coefficient }\end{array}$ \\
\hline DC Bead (confocal) & $87 \pm 13$ & 2.18 & 0.230 & 0.106 & 0.982 \\
LUMl (confocal) & $107 \pm 17$ & 0.75 & 0.090 & 0.120 & 0.905 \\
DC Bead (sectioning) & $87 \pm 13$ & 2.18 & 0.042 & 0.019 & 0.994 \\
LUMl (sectioning) & $107 \pm 17$ & 0.75 & 0.017 & 0.022 & 0.983 \\
\hline
\end{tabular}


* The data were presented in average size \pm standard deviation

** Average bead diameter was used for total bead volume calculation. Value 0.74 was used as bead packing factor.

\subsection{Comparison between in vitro - in vivo drug distribution}

It is of interest to compare the in vitro Dox distribution to in vivo data in order to better understand drug distribution and metabolism in tissue. Previous data from an in vivo study [10] were first compared with the current in vitro data (see Fig. S6). Photographs of bead-packed channels are included to show the halo effect of Dox distribution in both media. The drug distribution profile in the in vitro model extends over a greater distance $(>2 \mathrm{~mm})$ and indicates higher drug concentrations than in the in vivo data. Using Eq. 5 to estimate the in vivo drug concentration, indicated no more than $0.0026 \mathrm{mg} / \mathrm{mL}$ of drug around the channel, which is about 100 times lower than the drug in in vitro case $(\sim 0.25 \mathrm{mg} / \mathrm{mL})$. It is noted that the size of the two channels were not the same, in vivo the diameter was $\sim 500 \mu \mathrm{m}$, and in vitro it was $1600 \mu \mathrm{m}$, which means the in vitro volume of beads was $\sim 10$ times higher than that in vivo if the same length of channel was used for calculation. This cannot therefore explain the large difference between the two profiles, and hence the metabolism of Dox in tissue must have contributed to the lower drug concentration observed in vivo.

To further examine the effect of elimination on the concentration distribution of Dox around blood vessel, the Dox diffusion model (Eq. 1) was used to analyse the in vivo data [10], where $k \neq 0$. The fitted data with elimination effect and calculated data without considering elimination were both presented with in vivo data together in Fig. 5. Compared to the in vitro data (Fig. 2 and 3), the penetration distance of Dox in tissue was much shorter due to the cellular uptake and metabolism of the drug [30]. The estimated diffusivities $D$ were in the range of $0.6 \times 10^{-7}$ to $3.7 \times 10^{-7} \mathrm{~cm}^{2} \mathrm{~s}^{-1}$ within $24 \mathrm{hr}$ period. A fixed elimination coefficient [31] of $k=9.6 \times 10^{-4} \mathrm{~s}^{-1}$ was used in the calculation (see Table S5 in Supplementary Information) to avoid a strong correlation between diffusivity and elimination coefficient, which was observed during data fitting. The fitted diffusivities are close to the literature in vivo data $\left(5.8 \times 10^{-7}\right.$ to $\left.8.2 \times 10^{-7} \mathrm{~cm}^{2} \mathrm{~s}^{-1}\right)[31]$.

Based on the estimation above, a hypothesized profile of Dox diffusion without considering elimination effect could be constructed under the assumption that the diffusivities were maintained the same (dotted lines in Fig. 5). The amount of the drug eliminated could be calculated, which was assumed equivalent to the difference between the integration of solid line and dotted line. Subsequently the cumulative drug elution was obtained by removing the residual drug not consumed from the beginning in each distribution profile (the insert in Fig. 5D). It was observed that after $8 \mathrm{hr}$ the Dox release reached a plateau with only a slight increase. The calculated result is consistent with the images showed in Fig. E2 in the literature [10] 
477 where the beads had almost released all the payload at $24 \mathrm{hr}$. Based on the data 478 points, the dose released to the surrounding tissue is preliminarily estimated no less 479 than $7.2 \mathrm{mg} / \mathrm{mL}$ at $8 \mathrm{hr}$ and $7.4 \mathrm{mg} / \mathrm{mL}$ beads at $24 \mathrm{hr}$, which are about $1 / 5$ of the 480 drug loading $(37.5 \mathrm{mg} / \mathrm{mL}$ beads).

481
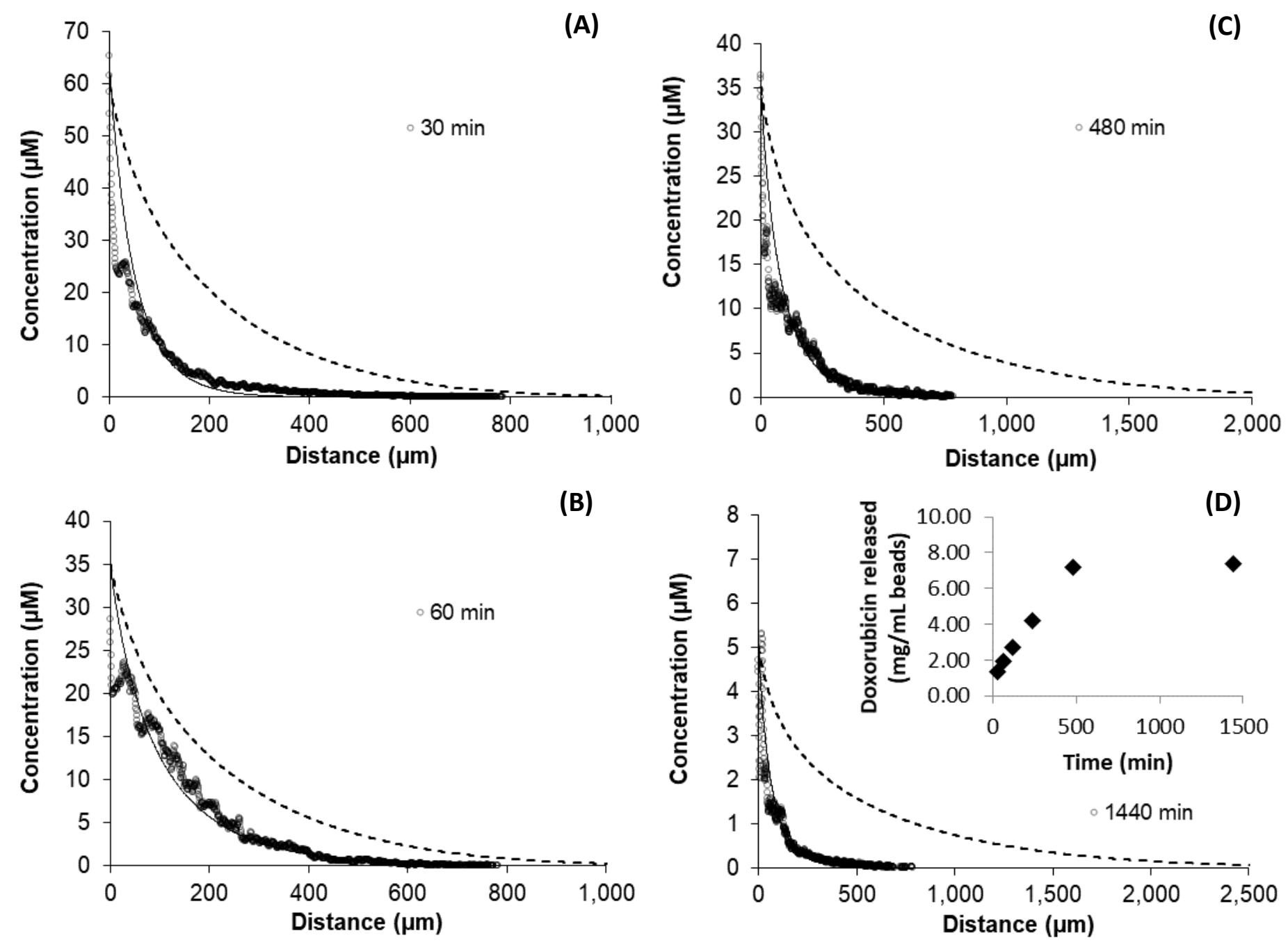

491

492 Figure 5. Fitting results (solid lines) of in vivo fluorescence data of Dox concentration 493 distribution at (A) $30 \mathrm{~min},(B) 60 \mathrm{~min},(C) 480 \mathrm{~min}$ and (D) $1440 \mathrm{~min}$, by using equation (1). 494 The dashed lines are the calculation of Dox concentration without elimination effect considered. The insert in Figure (D) is the accumulation of released drug calculated from the concentration distribution at different time point. Diffusivity was fitted with the boundary Dox concentration taking from the values estimated from the largest concentration in in vivo data and a fixed elimination coefficient value $k=9.6 \times 10^{-4} \mathrm{~s}^{-1}$ was used.

\subsection{Discussion}

When comparing the two techniques, the direct sectioning method is relatively 504 
values reported in in vivo studies and the literature [30, 31], and it provides direct information of Dox distribution with minimal impact of gel transparency at least in relatively thin samples. Its main limitations are the necessity for sample destruction during sectioning, and the fact that measurement accuracy can be affected by gel deformation during sectioning, e.g. inaccurate sectioning leading to non-uniform gel thickness, and Dox contamination on the gel surface from the blade smearing across the drug-containing lumen. The confocal fluorescence microscopy technique provides non-destructive measurements of Dox diffusion in situ in both radial and axial directions simultaneously. Similar methods have been applied to the analysis of drug distribution in tumour tissue, extracellular matrix and interstitial spaces [10, 32, 33]. When compared to direct sectioning method, key advantages of the method are its efficiency and high imaging rate that is particularly important in recording cases of fast drug diffusion. Another point is its convenience in measuring 3-dimensional drug distribution in the gel phase without laborious manual sectioning. The confocal method has also indicated higher accuracy when comparing diffusivities to historical in vivo data in terms of quantification and prediction due potentially to its inherently higher imaging accuracy and resolution. Limitations include the need for optical transparency to achieve good signal to noise rations, as the method requires optical imaging through the hydrogel thickness. This places a restriction on the path length for measurement (which equates to gel thickness) and on gel formulations that allow transmission of the light. The sectioning method on the other hand, measures drug distribution along the sectioned surface and thus has no such restrictions. The confocal method also requires bespoke software and system calibration, which also comes at greater expense and reduced accessibility.

In terms of inherent method differences, fluorescence microscopy floods the entire specimen in light and all parts of the specimen in the optical path are excited at the same time, the detected fluorescence therefore including a large unfocused background contribution. This means the signal could be sufficiently high to undergo fluorescence quenching, reducing the measured signal and producing an artificially low measurement for the sectioning method at the point of high drug concentration at the boundary. In contrast, the confocal method uses point illumination and a pinhole in an optically conjugate plane in front of the detector to eliminate out-of-focus signal. This means the image's optical resolution, particularly in the sample depth direction, is much better than that of wide-field microscopes giving a truer representation of the higher drug concentration at the boundary. The region of lower concentration values observed using the sectioning method close to the boundary is about 200-300 $\mu \mathrm{m}$ thick, which might be therefore be attributed to a non-uniform boundary effect and/or fluorescence quenching.

The results from the confocal fluorescence measurements were qualitatively very similar to those described above for the sectioning method. Dox penetration in a hydrogel composed of agarose $(1.5 \%)$-alginate $(0.5 \%)$ showed that the distribution area of Dox from DC Bead was larger than that of LUMI at equivalent time points 
(Fig. 3A-F). The total fluorescence measurement also confirms that DC Bead releases Dox faster than LUMI. The fluorescence intensities in the middle range were converted into concentration values. The concentration profiles derived from the confocal images indicated that at $150 \mathrm{~min}$ the penetration of Dox into the agarose-alginate gel was approximately $4000 \mu \mathrm{m}$ from the DC Bead packed channels, and $3000 \mu \mathrm{m}$ in models with LUMI (Fig. $3 \mathrm{G}-\mathrm{H}$ and compared with Fig. 2 $\mathrm{G}-\mathrm{H})$. The drug concentration of DC Bead read from the interface between beads and gel is about $1000 \mu \mathrm{M}$, compared to the value of $700 \mu \mathrm{M}$. This is consistent with the fact that DC Bead elutes Dox faster than an equivalent size range of LUMI, and the same effect was confirmed using either technique [6, 34].

When looking at the results from the different bead types using both methods, it can be seen that the rate of Dox release from DC Bead is faster than that from LUMI in these in vitro systems, which is due to the less dense hydrogel structure of DC Bead compared to the more compact and iodine-containing LUMI. Unloaded DC Bead contains about 95\% water and about 5\% PVA and poly(acrylamido-2methylpropanesulfonate, AMPS) network [7, 35]. In contrast LUMI contains about $45 \%$ polymer network comprising of PVA, polyAMPS and iodine moieties (the latter providing the radiopacity) [34]. Introducing hydrophobic iodinated moieties into beads generates further hydrophobic-hydrophobic interaction and formation of a physically tighter structure. The more compact structure significantly retards the diffusion of ions inside the gel and limits the ion exchange process, which leads to slow drug elution from beads into gel. The diameter of DC Bead also decreases in size after loading with Dox because of drug-drug interaction through the $\pi-\pi$ stacking of anthracycline ring of Dox and expulsion of water from the gel matrix [5, 7, 35, 36]. Water content, however, is still relatively high and the interstitial spaces between polymer chains remains large enough that Dox diffusion from the matrix is relatively unimpeded. A lower boundary drug concentration was observed in the case of LUMI therefore, as measured by both techniques, confirming the elution results obtained using conventional drug dissolution methods $[5,6]$ and the above theory of existing hydrophobic interactions.

When considering the treatment of liver tumours using DEBs, in addition to the physicochemical characteristics of the drug loaded in the beads, other factors such as vascular density, permeability, and the extent and density of the extracellular matrix, have direct impact on the efficacy of treatment [8, 30]. An effective phantom system is required to not only mimic appropriate properties of tumour extravascular tissue, but also needs to meet certain minimum performance criteria for the support of quality assurance and future regulatory approval [37]. Materials frequently used in polymeric phantoms include, for instance, agarose, polyacrylamide, PVA and gelatin. A match of biomechanical properties of tumour tissue with mimicking gel in studies would provide useful information for understanding drug diffusion and distribution in cancer treatment. The stiffness of the extracellular matrix is a key characteristic of tumor microenvironment which are composed of collagen type I [38]. The density of 
ECM in tumor increases when new ECM secretion and upregulation of cross-linkers and cancer cells. It has been demonstrated that 3D matrices with a Young's modulus of $400 \mathrm{~Pa}$ significantly increase the size of colonies compared to matrices with Young's modulus of 169-170 $\mathrm{Pa}$ [39]. In another study, it showed that the storage modulus of representative $4 \mathrm{~T} 1$ mouse model of mammary carcinoma and SW480 subcutaneous model of colorectal cancer are $3169 \pm 377 \mathrm{~Pa}$ and $3092 \pm 274$ $\mathrm{Pa}$, respectively, compared to normal tissue $161.9 \pm 20.4 \mathrm{~Pa}$ and SW480 cell line $1625 \pm 149.7 \mathrm{~Pa}$, respectively [40]. Agarose gel is composed of fibrillar networks formed by bundles of single- and double-helices, and at $20{ }^{\circ} \mathrm{C}$, a typical storage modulus of $1.0 \%$ of agarose gel were reported around $2000 \mathrm{~Pa}$ [41] to $10000 \mathrm{~Pa}$ [42], depending on the source and conditions of preparation. It has been used as phantoms to mimic liver tumor for elasticity and chogenicity analysis [43].

A combination of alginate and agarose gel (1.5\%) was used to provide suitable mechanical stiffness and potential binding sites for Dox with the gel, to better mimic drug interactions with tissue, although typical tissue contains $77 \%$ water and the agarose gel contains $98 \%$ water. Despite a relatively weak interaction between Dox and alginate $[18,44]$, the current result from the measurement by both techniques suggested that the selected alginate: agarose formulation was useful as a tissue mimicking hydrogel. Different alginate concentration from $0 \%, 0.5 \%$ to $1.0 \%$ were tested to explore the interaction between Dox and hydrogel components. The drug diffusion profiles showed no significant difference (Section S3), indicating that the selected concentrations of alginate were not high enough to affect Dox diffusion behaviour. The diffusivities of Dox obtained from the tissue-mimicking alginateagarose hydrogel in the range between $6.07 \times 10^{-7}$ to $1.33 \times 10^{-6} \mathrm{~cm}^{2} \mathrm{~s}^{-1}$ (Table 1) were slightly higher than in vivo values $\left(0.6 \times 10^{-7}\right.$ to $3.7 \times 10^{-7} \mathrm{~cm}^{2} \mathrm{~s}^{-1}$ [Section 3.3] and $5.8 \times 10^{-7}$ to $8.2 \times 10^{-7} \mathrm{~cm}^{2} \mathrm{~s}^{-1}$ [31]) which suggests a further tuning of gel composition may needed to decrease Dox diffusivities for matching the in vivo data. From methodology point, the results suggest that both the in vitro confocal microscopy method and gel formulation provided a promising test method to measure the correlation between in vitro and in vivo drug release properties of DEB especially in a microscopic level. One drawback that remains however, is that it is not obvious as to how to physically include a drug elimination process akin to that which occurs within the tissue, other than modelling the process and extrapolating the data to include an elimination factor as we have done here. Additional mechanisms to eliminate the drug by either extra physical diffusion/convection, chemical binding or biological metabolism could be included in future study.

During the process of Dox release and diffusion into the hydrogel phase, there are several processes of equilibrium involved. Dox is initially bound to the bead via ionic interaction between positively charged drug and the negatively charged sulfonate groups on the bead structure. In addition to this, the self-association of Dox molecules occurs when in close proximity to one another within the bead structure. 
This may lead to the formation of larger aggregate structures from dimers, trimers, multimers to fibres [36]. Once drug is displaced from the bead structure by competitive ion displacement, the drug diffuses through the matrix and into the hydrogel mimic where there is a binding equilibrium between Dox and carboxylic acid groups on alginate.

Fig. 5 shows a direct comparison between in vitro and in vivo data, with the Dox elution from DC Bead in vitro (phantom gel sectioning method) and in vivo (data extracted from a published study of embolised tissue, no radiopaque LUMI product was available for in vivo analysis as this was prior to development and launch), which suggests that the drug release from a large vessel packed with DEBs is slower compared to the beads packed in a small size vessel. This is consistent with the fact that the equivalent volumes of beads within multiple small vessels versus one large vessel, will possess a larger surface area, with more beads contacting the lumen wall, leading to a faster release of drug. This is somewhat akin to the use of the classic USP II dissolution method to mimic release from multiple small vessels, as drug is extracted from the beads by suspension in a large volume of eluent. Recently additional custom elution devices have been described that better mimic the unique flow mechanics, diffusion and convection process experienced during embolic occlusion in larger vessels [5-7]. However, these flow models fail to capture the critical contact diffusion characteristics as investigated in the current investigation. Either the physical sectioning, or confocal methods described herein have the distinct advantage of allowing the flexibility of selecting vessel lumen size to be studied. Vessel size is a one of the main factors to consider in the design of DEBs, and smaller size beads have been demonstrated to penetrate deeper into tumour vessels and cause more tissue necrosis [9]. Clinical benefits of smaller size beads have been demonstrated in human trials $[2,8]$. Here we only evaluated release from beads of size 70-150 $\mu \mathrm{m}$ but studies could be extended to evaluate the effect of bead size on drug elution phenomena in tissue mimicking phantoms. Moreover, although in this study we restricted the phantom composition to one formulation of alginate: agarose, further studies could investigate the impact of composition, or indeed the effect of different gel-forming materials, on drug release kinetics.

\section{Conclusions}

In this study two novel methods were used to monitor drug elution in a hydrogel tissue-mimic. The results demonstrated qualitatively comparable trends to historical in vivo data. There was an approximately one order of magnitude difference in the measured Dox distribution measured between the physical sectioning and real-time confocal methods. This and other discrepancies observed in boundary drug concentration and diffusion distance were attributed to differences in sample preparation and quenching effects between the two methods. 
The observed elution rate variations between different bead types illustrate the sensitivity of both methods for tissue distribution determination and demonstrates their utility for enabling the design and optimisation of DEB systems. The confocal method offers higher efficiency and temporal resolution at the cost of flexibility and expense; whilst physical sectioning provides a more painstaking baseline method for predictive modelling. A future combined approach as part of further method and gel material validation will surely yield the most effective method of predictive drug release profiling in vitro whilst removing the need for further costly, laborious and unethical animal models.

681

\section{Acknowledgements}

SB would like to thank the Royal Commission for the Exhibition of 1851 for provision of an Industrial Fellowship. ES would like to thank the Engineering and Physical Sciences Research Council (EP/I021795/1 and EP/L024012/1) and the Institute of Engineering and Technology (AF Harvey Prize) for funding. DD and ES would like to thank the NC3Rs Crack It Solutions Scheme for funding.

\section{Declaration of interest}

No authors have any relevant disclosures.

\section{References}

1. Varela, M., et al., Chemoembolization of hepatocellular carcinoma with drug eluting beads: efficacy and doxorubicin pharmacokinetics. J Hepatol, 2007. 46(3): p. 474-81.

2. Prajapati, H.J., et al., Survival, efficacy, and safety of small versus large doxorubicin drugeluting beads TACE chemoembolization in patients with unresectable HCC. AJR Am J Roentgenol, 2014. 203(6): p. W706-14.

3. Malagari, K., et al., Pharmacokinetics, Safety, and Efficacy of Chemoembolization with Doxorubicin-Loaded Tightly Calibrated Small Microspheres in Patients with Hepatocellular Carcinoma. Cardiovasc Intervent Radiol, 2016. 39(10): p. 1379-91.

4. Hwang, C.-W. and E.R. Edelman, Arterial Ultrastructure Influences Transport of Locally Delivered Drugs. Circulation Research, 2002. 90(7): p. 826-832.

5. Swaine, T., et al., Evaluation of ion exchange processes in drug-eluting embolization beads by use of an improved flow-through elution method. European Journal of Pharmaceutical Sciences, 2016. 93: p. 351-359.

6. Hagan, A., et al., Predicting pharmacokinetic behaviour of drug release from drug-eluting embolization beads using in vitro elution methods. European Journal of Pharmaceutical Sciences, 2019.

7. Gonzalez, M.V., et al., Doxorubicin eluting beads-2: methods for evaluating drug elution and in-vitro:in-vivo correlation. J Mater Sci Mater Med, 2008. 19(2): p. 767-75.

8. Namur, J., et al., Embolization of hepatocellular carcinoma with drug-eluting beads: doxorubicin tissue concentration and distribution in patient liver explants. J Hepatol, 2011. 55(6): p. 1332-8.

9. Namur, J., et al., Drug-eluting beads for liver embolization: concentration of doxorubicin in tissue and in beads in a pig model. J Vasc Interv Radiol, 2010. 21(2): p. 259-67. 
10. Dreher, M.R., et al., Radiopaque drug-eluting beads for transcatheter embolotherapy: experimental study of drug penetration and coverage in swine. J Vasc Interv Radiol, 2012. 23(2): p. 257-64 e4.

11. Hu C, C.Y., Tan MJA, Ren K, Wu H, Microfluidic technologies for vasculature biomimicry Analyst, 2019. 144(15): p. 4461-4471.

12. Kim, D., et al., Microfluidics-based in vivo mimetic systems for the study of cellular biology. Acc Chem Res, 2014. 47(4): p. 1165-73.

13. Carugo, D., et al., A microfluidic device for the characterisation of embolisation with polyvinyl alcohol beads through biomimetic bifurcations. Biomed Microdevices, 2012. 14(1): p. 15363.

14. Caine, M., et al., Impact of Yttrium-90 Microsphere Density, Flow Dynamics, and Administration Technique on Spatial Distribution: Analysis Using an In Vitro Model. J Vasc Interv Radiol, 2017. 28(2): p. 260-268 e2.

15. Hagan, A., et al., Predicting pharmacokinetic behaviour of drug release from drug-eluting embolization beads using in vitro elution methods. Eur J Pharm Sci, 2019. 136: p. 104943.

16. Yanan Gao, Z.L., Yin Hong, Tingting Li, Xiaoyan Hu, Luyao Sun, Zhengchang Chen, Zijian Chen, Zhiheng Luo, Xin Wang, Jian Kong, Guanglei Li, Hsing-Lin Wang, Hwa Liang Leo, Hanry Yu, Lei $\mathrm{Xi}$, Qiongyu Guo,, Decellularized liver as a translucent ex vivo model for vascular embolization evaluation. Biomaterials, 2020. 240: p. 119855.

17. Zhang, X., L. Li, and C. Luo, Gel integration for microfluidic applications. Lab on a Chip, 2016. 16(10): p. 1757-1776.

18. van Elk, M., et al., Alginate Microspheres Containing Temperature Sensitive Liposomes (TSL) for MR-Guided Embolization and Triggered Release of Doxorubicin. PLoS One, 2015. 10(11): p. e0141626.

19. Shuning Bian, A.S., Dan Daly, Robert Carlisle, and Eleanor Stride, A multimodal instrument for real-time in situ study of ultrasound and cavitation mediated drug delivery. Review of Scientific Instruments 2017. 88(3): p. 034302.

20. Biondi, M., et al., Investigation of the mechanisms governing doxorubicin and irinotecan release from drug-eluting beads: mathematical modeling and experimental verification. J Mater Sci Mater Med, 2013. 24(10): p. 2359-70.

21. Lee, K.H., et al., Doxorubicin-loaded QuadraSphere microspheres: plasma pharmacokinetics and intratumoral drug concentration in an animal model of liver cancer. Cardiovasc Intervent Radiol, 2010. 33(3): p. 576-82.

22. Cheung, R.Y., et al., A new approach to the in vivo and in vitro investigation of drug release from locoregionally delivered microspheres. J Control Release, 2004. 100(1): p. 121-33.

23. Lewis, A.L., et al., DC bead: in vitro characterization of a drug-delivery device for transarterial chemoembolization. J Vasc Interv Radiol, 2006. 17(2 Pt 1): p. 335-42.

24. Li, Y., A.M. Rauth, and X.Y. Wu, Prediction of kinetics of doxorubicin release from sulfopropyl dextran ion-exchange microspheres using artificial neural networks. Eur J Pharm Sci, 2005. 24(5): p. 401-10.

25. Sawaya, A., et al., lon-exchange albumin microcapsules of doxorubicin: an in vitro release kinetic evaluation. J Microencapsul, 1988. 5(3): p. 255-67.

26. Panos Macheras, A.I., Modeling in Biopharmaceutics, Pharmacokinetics, and PHarmacodynamics: Homogeneous and Heterogeneous Approaches. 2006, New York: Springer.

27. Liu, Z., et al., A study of doxorubicin loading onto and release from sulfopropyl dextran ionexchange microspheres. J Control Release, 2001. 77(3): p. 213-24.

28. Dreher, M.R., et al., Radiopaque Drug-Eluting Beads for Transcatheter Embolotherapy: Experimental Study of Drug Penetration and Coverage in Swine. Journal of Vascular and Interventional Radiology, 2012. 23(2): p. 257-264.e4. 
29. Amyot, F., et al., A new experimental method for the evaluation of the release profiles of drug-loaded microbeads designed for embolisation. ITBM-RBM, 2002. 23(5): p. 285-289.

30. Dewhirst, M.W. and T.W. Secomb, Transport of drugs from blood vessels to tumour tissue. Nat Rev Cancer, 2017. 17(12): p. 738-750.

31. Feng Qian, N.S., Erin H. Liu , Gerald M. Saidel , Jinming Gao, Quantification of in vivo doxorubicin transport from PLGA millirods in thermoablated rat livers. Journal of Controlled Release, 2003. 91: p. 157-166.

32. Khalid, A., et al., Doxorubicin loaded nanodiamond-silk spheres for fluorescence tracking and controlled drug release. Biomed Opt Express, 2016. 7(1): p. 132-47.

33. Catharina de L. Davies, L.M.L., Jomar Frengen, Live Eikenes, Øyvind S. Bruland, Olav Kaalhus, Mari H. B. Hjelstuen, and Christian Brekken, Radiation Improves the Distribution and Uptake of Liposomal Doxorubicin (Caelyx) in Human Osteosarcoma Xenografts. CANCER RESEARCH 2004. 64: p. 547-553

34. Koorosh Ashrafi, Y., Hugh Britton, Orianne Domenge, Delphine Blino, Andrew J. Bushby, Kseniya Shuturminska, Mark den Hartog, Alessandro Radaelli, Ayele H. Negussie, Andrew S.Mikhail, David L.Woods, Venkatesh Krishnasamy, ElliotB. Levy, Bradford J.Wood, Sean L.Willis, MatthewR. Dreher, AndrewL. Lewis, Characterization of a novel intrinsically radiopaque Drug-eluting Bead for image-guided therapy: DC Bead LUMI ${ }^{T M}$. Journal of Controlled Release, 2017. 250: p. 36-47.

35. Lewis, A.L., et al., Doxorubicin eluting beads - 1: effects of drug loading on bead characteristics and drug distribution. J Mater Sci Mater Med, 2007. 18(9): p. 1691-9.

36. Li, X., et al., Doxorubicin physical state in solution and inside liposomes loaded via a $\mathrm{pH}$ gradient. Biochimica et Biophysica Acta (BBA) - Biomembranes, 1998. 1415(1): p. 23-40.

37. Pogue, B.W. and M.S. Patterson, Review of tissue simulating phantoms for optical spectroscopy, imaging and dosimetry. J Biomed Opt, 2006. 11(4): p. 041102.

38. Kyung Min Park, D.L., and Sharon Gerecht, Bioinspired Hydrogels to Engineer Cancer Microenvironments. Annu. Rev. Biomed. Eng., 2017. 19: p. 109-33.

39. Matthew J Paszek, N.Z., Kandice R Johnson, Johnathon N Lakins, Gabriela I Rozenberg, Amit Gefen, Cynthia A Reinhart-King, Susan S Margulies, Micah Dembo, David Boettiger, Daniel A Hammer, Valerie M Weaver Tensional homeostasis and the malignant phenotype. Cancer Cell, 2005. 8: p. 241-54.

40. Madsen, C. and T. Cox, Relative Stiffness Measurements of Tumour Tissues by Shear Rheology. Bio-Protocol, 2017. 7(9): p. e2265.

41. Bertula, K., et al., Strain-Stiffening of Agarose Gels. ACS Macro Letters, 2019. 8(6): p. 670675.

42. Fernández, E., et al., Rheological and thermal properties of agarose aqueous solutions and hydrogels. Journal of Polymer Science Part B: Polymer Physics, 2008. 46(3): p. 322-328.

43. Bin, L., Y. Ronghua, Y. Peng, M. Awad, M. Choti and R. Taylor, Elasticity and Echogenicity Analysis of Agarose Phantoms Mimicking Liver Tumors. Proceedings of the IEEE 32nd Annual Northeast Bioengineering Conference, 2006: p. 81-82.

44. Bosio, V.E., et al., Binding and encapsulation of doxorubicin on smart pectin hydrogels for oral delivery. Appl Biochem Biotechnol, 2012. 167(5): p. 1365-76. 